\title{
Turbo Warrants under Hybrid Stochastic and Local Volatility
}

\author{
Min-Ku Lee, ${ }^{1}$ Ji-Hun Yoon, ${ }^{2}$ Jeong-Hoon Kim, ${ }^{3}$ and Sun-Hwa Cho ${ }^{3}$ \\ ${ }^{1}$ Department of Mathematics, Sungkyunkwan University, Suwon-si, Gyeonggi-do 440-746, Republic of Korea \\ ${ }^{2}$ Department of Mathematical Science, Seoul National University, Seoul 151-747, Republic of Korea \\ ${ }^{3}$ Department of Mathematics, Yonsei University, Seoul 120-749, Republic of Korea \\ Correspondence should be addressed to Jeong-Hoon Kim; jhkim96@yonsei.ac.kr
}

Received 28 September 2013; Revised 12 December 2013; Accepted 14 December 2013; Published 8 January 2014

Academic Editor: Sergio Polidoro

Copyright (C) 2014 Min-Ku Lee et al. This is an open access article distributed under the Creative Commons Attribution License, which permits unrestricted use, distribution, and reproduction in any medium, provided the original work is properly cited.

\begin{abstract}
This paper considers the pricing of turbo warrants under a hybrid stochastic and local volatility model. The model consists of the constant elasticity of variance model incorporated by a fast fluctuating Ornstein-Uhlenbeck process for stochastic volatility. The sensitive structure of the turbo warrant price is revealed by asymptotic analysis and numerical computation based on the observation that the elasticity of variance controls leverage effects and plays an important role in characterizing various phases of volatile markets.
\end{abstract}

\section{Introduction}

Turbo warrants, which appeared first in Germany in late 2001, have experienced a considerable growth in Northern Europe and Hong Kong. They are special types of knockout barrier options in which the rebate is calculated as another exotic option. For one thing, this contract has a low vega so that the option price is less sensitive to the change of the implied volatility of the security market, and for another, it is highly geared owing to the possibility of knockout. Closed form expression for the price has been presented by Eriksson [1] under geometric Brownian motion (GBM) for the underlying security.

It is well-known that the assumption of the GBM for the underlying security price in the Black-Scholes model [2] does not capture many empirical lines of evidence appeared in financial markets. Maybe, the two most significant shortcomings of the model's assumption lie in flat implied volatility, whereas the volatility fluctuates depending upon market conditions and the underestimation of extreme moves, yielding tail risk. So, there have been a lot of alternative underlying models developed to extend the GBM and overcome these problems. Local volatility models are one type of them, where the volatility depends on the price level of the underlying security itself. The most well-known local volatility model is the constant elasticity of variance $(\mathrm{CEV})$ model in which the volatility is given by a power function of the underlying security price. It has been proposed by Cox [3] and Cox and Ross [4]. Another version of volatility models has been developed by assuming security's volatility to be a random process governed by another state variables such as the tendency of volatility to revert to some long-run mean value, the variance of the volatility process itself, and so forth. This type of models is called (pure) stochastic volatility models. The models developed by Heston [5] and Fouque et al. [6] are popular ones among others in this category. Also, there is another type of generalized models for the underlying security which have been developed in terms of Levy processes which may have discontinuous paths. The model by Carr et al. [7] is a well-known one of this kind among others.

Even if those models have been developed to overcome the major shortcomings of the Black-Scholes model, there still remain limitations. For instance, volatility and underlying risky asset price changes are perfectly correlated either positively or negatively in the CEV model, whereas empirical studies indicate no definite correlation all the time. Also, the dynamics of the implied volatility surface created by the CEV model may have an opposite direction to the observed market dynamics as shown by Hagan et al. [8]. Stochastic volatility models usually do not take into account the relationship between volatility and underlying price (the leverage or inverse leverage effects) directly. Much of computing time 
tends to be required for the implementation of Levy type of models. So, recently, one has begun to see a trend of developing some sort of hybrid formulation in asset pricing. A hybrid stochastic and local volatility model created by Choi et al. [9] is among those alternative hybrid models for pricing options. This new hybrid model is equipped with the stochastic volatility and CEV terms in the volatility part. From the hybrid nature of the volatility, one can remove the hedging instability created by the CEV model as well as capture the leverage effect fit to the corresponding market. To date, there have been a number of applications of this hybrid model to the pricing of derivatives. Refer to Choi et al. [9] and Bock et al. [10] for European options and Lee et al. [11] for Asian options.

This paper is concerned with the pricing of turbo warrants which has been studied by several authors for different models of security price in recent years. The pricing of turbo warrants has been studied by Eriksson [1] under the Black-Scholes model, Wong and Chan [12] under a stochastic volatility model, Domingues [13] under the CEV model, and Wong and Lau [14] under a jump diffusion model. This work studies the pricing of a turbo warrant under the hybrid setting of stochastic and local volatility provided by [9]. In particular, the main concern is given to the sensitivity of the turbo warrant to the elasticity parameter and parameters driving the stochastic volatility. The choice to price a turbo warrant with this kind of hybrid model is justified as follows. Turbo warrants are barrier options with the rebate whose value is computed by another path dependent option. They can be very sensitive to the change in volatility under stochastic volatility models as pointed out by [12], which is contrary to the case of the Black-Scholes model. However, a frequent criticism of stochastic volatility models for path dependent options is that they do not produce deltas precise enough for hedging purposes. So, relevant industry experts recommend using a hybrid stochastic local volatility model of their own development for the best pricing of option products. Refer to Tataru and Travis [15]. Since the elasticity of variance controls leverage effects and plays an important role in characterizing various phases of volatile markets as described in Kim et al. [16], it is worth investigating the sensitivity of the price to elasticity parameter under a hybrid structure of the stochastic volatility and the constant elasticity of variance.

This paper is structured as follows. Section 2 provides a review on the decomposition of the price of turbo warrants call option and establish a partial differential equation (PDE) for the price based on the hybrid model (called the SVCEV model) of [9]. In Section 3, asymptotic analysis is given for the PDE under the assumption of fast mean reverting stochastic volatility. Section 4 solves the approximate price by using the finite difference method and investigates the price behavior and compares it with the ones corresponding to a stochastic volatility model as well as the CEV model. Section 5 concludes.

\section{Model Formulation}

The contract concerned in this paper is defined as follows. A turbo call warrant for an underlying asset, whose price at time $t$ is given by $S_{t}$, pays the option possessor $\left(S_{T}-K\right)^{+}$at maturity $T$ on the understanding that a specified barrier $H \geq$ $K$ has not been passed by $S_{t}$ at any time prior to the maturity. Let us define $\tau_{H}$ as the first time that the asset price hits the barrier $H$, that is, $\tau_{H}=\inf \left\{t \mid S_{t} \leq H\right\}$. The contract is useless and a new contract begins if $\tau_{H} \leq T$. The new contract is a call option whose payoff is given by the difference between $m_{\tau_{H}}^{T_{0}}:=\min _{\tau_{H} \leq t \leq \tau_{H}+T_{0}} S_{t}$ and the strike price $K$ with time to maturity $T_{0}$. Thus the turbo call contract is given by

$$
\begin{aligned}
\operatorname{TC}(t, s)= & E\left[e^{-r(T-t)}\left(S_{T}-K\right)^{+} \mathbf{1}_{\left\{\tau_{H}>T\right\}} \mid S_{t}=s\right] \\
& +E\left[e^{-r\left(\tau_{H}+T_{0}-t\right)}\left(m_{\tau_{H}}^{T_{0}}-K\right)^{+} \mathbf{1}_{\left\{\tau_{H} \leq T\right\}} \mid S_{t}=s\right],
\end{aligned}
$$

where $E$ denotes the expectation with respect to a riskneural probability. The turbo call warrant denoted by (1) is comprised of two parts. The first part looks like a down-andout barrier (DOC) call option with a zero rebate and the second part is a down-and-in lookback (DIL) call option. So, the price of each part is expressed as follows:

$$
\begin{gathered}
\operatorname{DOC}(t, s)=E\left[e^{-r(T-t)}\left(S_{T}-K\right)^{+} \mathbf{1}_{\left\{\tau_{H}>T\right\}} \mid S_{t}=s\right], \\
\operatorname{DIL}\left(t, s, T_{0}\right)=E\left[e^{-r\left(\tau_{H}+T_{0}-t\right)}\left(m_{\tau_{H}}^{T_{0}}-K\right)^{+} \mathbf{1}_{\left\{\tau_{H} \leq T\right\}} \mid S_{t}=s\right] .
\end{gathered}
$$

If $\operatorname{LB}\left(\tau_{H}, S_{\tau_{H}}, T_{0}\right):=E_{\tau_{H}}\left[e^{-r T_{0}}\left(m_{\tau_{H}}^{T_{0}}-K\right)^{+}\right]$(nonstandard lookback option) and $\mathrm{LC}_{\mathrm{fl}}(t, s, m, T)$ denotes the price of the floating strike lookback call on $S_{t}=s$ with realized minimum $m$ and time to maturity $T$, then one can obtain the following result.

Theorem 1. At $t<\tau_{H}$, the model-free representation of the turbo call warrant is given by

$T C(t, s)$

$$
\begin{aligned}
& =\operatorname{DOC}(t, s)+E\left[e^{-r\left(\tau_{H}-t\right)} \mathbf{1}_{\left\{\tau_{H} \leq T\right\}} L B\left(\tau_{H}, S_{\tau_{H}}, T_{0}\right) \mid S_{t}=s\right] \\
& =\operatorname{DOC}(t, s)+E\left[e^{-r\left(\tau_{H}-t\right)} \mathbf{1}_{\left\{\tau_{H} \leq T\right\}} L B\left(\tau_{H}, H, T_{0}\right) \mid S_{t}=s\right] \\
& =\operatorname{DOC}(t, s)+L B\left(\tau_{H}, S_{\tau_{H}}, T_{0}\right) E\left[e^{-r\left(\tau_{H}-t\right)} \mathbf{1}_{\left\{\tau_{H} \leq T\right\}} \mid S_{t}=s\right],
\end{aligned}
$$

where

$$
\begin{aligned}
L B\left(\tau_{H}, S_{\tau_{H}}, T_{0}\right)= & L C_{f l}\left(\tau_{H}, S_{\tau_{H}}, \min \left(S_{\tau_{H}}, K\right), T_{0}\right) \\
& -L C_{f l}\left(\tau_{H}, S_{\tau_{H}}, S_{\tau_{H}}, T_{0}\right) .
\end{aligned}
$$

In particular, if $S_{t}=H\left(\right.$ i.e., $\left.t=\tau_{H}\right)$, then

$$
T C(t, H)=E_{\tau_{H}}\left[e^{-r T_{0}}\left(m_{\tau_{H}}^{T_{0}}-K\right)^{+}\right]=L B\left(\tau_{H}, S_{\tau_{H}}, T_{0}\right) .
$$

Proof. Refer to Domingues [13]. 
This section constructs a pricing problem for turbo warrants based upon a hybrid stochastic and local volatility model given by [9]. Let $S_{t}$ be the underlying asset price at time $t$ and $W_{t}^{*}$ and $Z_{t}^{*}$ independent Brownian motions. Then the dynamics of $S_{t}$ are given by

$$
\begin{gathered}
d S_{t}=(r-q) S_{t} d t+f\left(Y_{t}\right) S_{t}^{\theta / 2} d W_{t}^{*}, \\
d Y_{t}=\left[\frac{1}{\epsilon}\left(m-Y_{t}\right)-\frac{1}{\sqrt{\epsilon}} \Lambda\left(Y_{t}\right)\right] d t+\frac{1}{\sqrt{\epsilon}} d \widehat{Z}_{t}^{*}
\end{gathered}
$$

under an equivalent martingale measure $Q^{\gamma}$ with an arbitrary adapted process $\gamma$ to be determined, where $r$ (interest rate), $q$ (dividend yield rate), $\theta$ (elasticity parameter), $\epsilon$ (reciprocal of rate of mean reversion), and $m$ are constants and $\widehat{Z}_{t}^{*}=\rho W_{t}^{*}+$ $\sqrt{1-\rho^{2}} Z_{t}^{*},-1 \leq \rho \leq 1$, and $\Lambda$ (market price of volatility risk) is independent of $S_{t}$, and the function $f$ is assumed to satisfy $0<c_{1}<f<c_{2}<\infty$ for some positive constants $c_{1}$ and $c_{2}$. Note that the Ornstein-Uhlenbeck $(\mathrm{OU})$ process $Y_{t}$ is a Gaussian process which has an invariant distribution given by $\mathcal{N}(m, 1 / 2)$. We call the model (6) and (7) the SVCEV model as in [9].

Now, under the SVCEV model above, the dependence of the value $Y_{t}=y$ is added to the price of the turbo warrant call option which yields

$$
\operatorname{TC}(t, s, y)=\operatorname{DOC}(t, s, y)+\operatorname{DIL}\left(t, s, y, T_{0}\right),
$$

where $\operatorname{DOC}(t, s, y)$ and $\operatorname{DIL}\left(t, s, y, T_{0}\right)$ are defined by (2) with the additional dependence of $y$, respectively. Also, $y$-dependence is added to $\mathrm{LB}$ and $\mathrm{LC}_{\mathrm{ff}} . \mathrm{LB}\left(H, y, T_{0}\right)=$ $\operatorname{LB}\left(\tau_{H}, S_{\tau_{H}}, y, T_{0}\right)$ and $\mathrm{LC}_{\mathrm{fl}}\left(H, y, T_{0}\right)=\mathrm{LC}_{\mathrm{fl}}\left(\tau_{H}, S_{\tau_{H}}, m, y, T_{0}\right)$ are used for briefness.

From now on, let $P(t, s, y):=\mathrm{TC}(t, s, y)$ for convenience. Then, by the well-known Feynman-Kac formula (cf. Øksendal [17]) on (8), $P(t, s, y)$ satisfies a PDE problem given by

$$
\begin{gathered}
\mathscr{L}^{\epsilon} P(t, s, y)=0, \quad 0 \leq t<T, x>0, \\
P(T, s, y)=s-K, \\
P\left(\tau_{H}, H, y\right)=\operatorname{LB}\left(\tau_{H}, H, y, T_{0}\right) .
\end{gathered}
$$

Here, if $\epsilon$ is positive and small, which corresponds to the assumption of the fast mean reversion of the process $Y_{t}$, then the operator $\mathscr{L}^{\epsilon}$ can be expressed by the following wellordered form:

$$
\begin{aligned}
& \mathscr{L}^{\epsilon}= \frac{1}{\epsilon} \mathscr{L}_{0}+\frac{1}{\sqrt{\epsilon}} \mathscr{L}_{1}+\mathscr{L}_{2}, \\
& \mathscr{L}_{0}:=\frac{1}{2} \partial_{y y}^{2}+(m-y) \partial_{y}, \\
& \mathscr{L}_{1}:=\rho f(y) s^{\theta / 2} \partial_{s y}^{2}-\Lambda(y) \partial_{y}, \\
& \mathscr{L}_{2}:=\partial_{t}+\frac{1}{2} f(y)^{2} s^{\theta} \partial_{s s}^{2} \\
&+(r-q) s \partial_{s}-r:=\mathscr{L}_{\mathrm{CEV}}(f(y)) .
\end{aligned}
$$

Note that the operator $(1 / \epsilon) \mathscr{L}_{0}$ is the infinitesimal generator (market probability measure version) of the OU process $Y_{t}$ and acts only on the $y$ variable. Since the operator $\mathscr{L}_{2}$ corresponds to the CEV operator for the turbo warrant, it is denoted by $\mathscr{L}_{\mathrm{CEV}}$ also.

\section{Approximation}

Solution of the PDE (9) is not analytically available. So, this section is devoted to value the turbo warrant by transforming (9) into a system of PDEs (under the assumption that $\epsilon$ is sufficiently small but positive) and solving the asymptotic PDEs numerically.

Under the assumption of the fast mean reversion of the process $Y_{t}$, this paper takes the asymptotic expansion,

$$
P=P_{0}+\sqrt{\epsilon} P_{1}+\epsilon P_{2}+\epsilon \sqrt{\epsilon} P_{3}+\cdots,
$$

and is interested in the first order approximation $P_{0}+\sqrt{\epsilon} P_{1}$. This work assumes a growth condition for each $P_{i}, i=$ $0,1,2, \ldots$, such that the derivative $\partial P_{i} / \partial y$ does not grow exponentially as much as $\left(\partial P_{i} / \partial y\right) \sim e^{y^{2} / 2}$ as $y \rightarrow \infty$.

First, a boundary condition on $P_{i}, i=0,1$, is provided as follows. Note that the first order approximation for the floating lookback call option $\mathrm{LC}_{\mathrm{fl}}$ is given by

$$
\operatorname{LC}_{\mathrm{fl}}\left(\tau_{H}, H, m, y, T_{0}\right) \approx \mathrm{LC}_{\mathrm{fl}}^{0}\left(H, T_{0}\right)+\sqrt{\epsilon} \mathrm{LC}_{\mathrm{fl}}^{1}\left(H, T_{0}\right),
$$

where $\operatorname{LC}_{\mathrm{fl}}^{0}\left(H, T_{0}\right)$ is the CEV price of $\mathrm{LC}_{\mathrm{fl}}$ with effective volatility $\bar{\sigma}^{2}=\left\langle f^{2}\right\rangle$, where $f$ is a function in (6) and $\operatorname{LC}_{\mathrm{fl}}^{1}\left(H, T_{0}\right)$ is the first corrected price of $\mathrm{LC}_{\mathrm{ff}}$. Here, $\mathrm{LC}_{\mathrm{ff}}^{0}\left(H, T_{0}\right)$ and $\mathrm{LC}_{\mathrm{fl}}^{1}\left(H, T_{0}\right)$ are independent of $y$ as in Wong and Chan [18]. By plugging (12) into $\operatorname{LB}\left(\tau_{H}, S_{\tau_{H}}, y, T_{0}\right)=$ $\operatorname{LC}_{\mathrm{fl}}\left(S_{\tau_{H}}, \min \left(S_{\tau_{H}}, K\right), y, T_{0}\right)-\mathrm{LC}_{\mathrm{fl}}\left(S_{\tau_{H}}, S_{\tau_{H}}, y, T_{0}\right)$, one can obtain the first order approximation:

$$
\begin{aligned}
P\left(\tau_{H}, H, y\right)= & \mathrm{LB}\left(\tau_{H}, H, y, T_{0}\right) \approx \mathrm{LB}_{0}\left(H, T_{0}\right) \\
& +\sqrt{\epsilon} \mathrm{LB}_{1}\left(H, T_{0}\right),
\end{aligned}
$$

where $\mathrm{LB}_{0}$ and $\mathrm{LB}_{1}$ are independent of $y$ and time $t$. Consequently, the boundary conditions

$$
\begin{gathered}
P_{0}(T, s, y)=s-K, \\
P_{1}(T, s, \mathrm{y})=0, \\
P_{i}\left(\tau_{H}, H\right)=\mathrm{LB}_{i}\left(H, T_{0}\right)
\end{gathered}
$$

are obtained for $P_{i}, i=0,1$.

The following lemma is extremely useful for the analysis of interest in this work.

Lemma 2. Let $\mathscr{L}_{0}$ be the operator given by (10). If solution to the Poisson equation

$$
\mathscr{L}_{0} \chi(y)+\phi(y)=0
$$

exists, then condition $\langle\phi\rangle=0$ must be satisfied, where $\langle\cdot\rangle$ denotes the expectation with respect to the invariant 


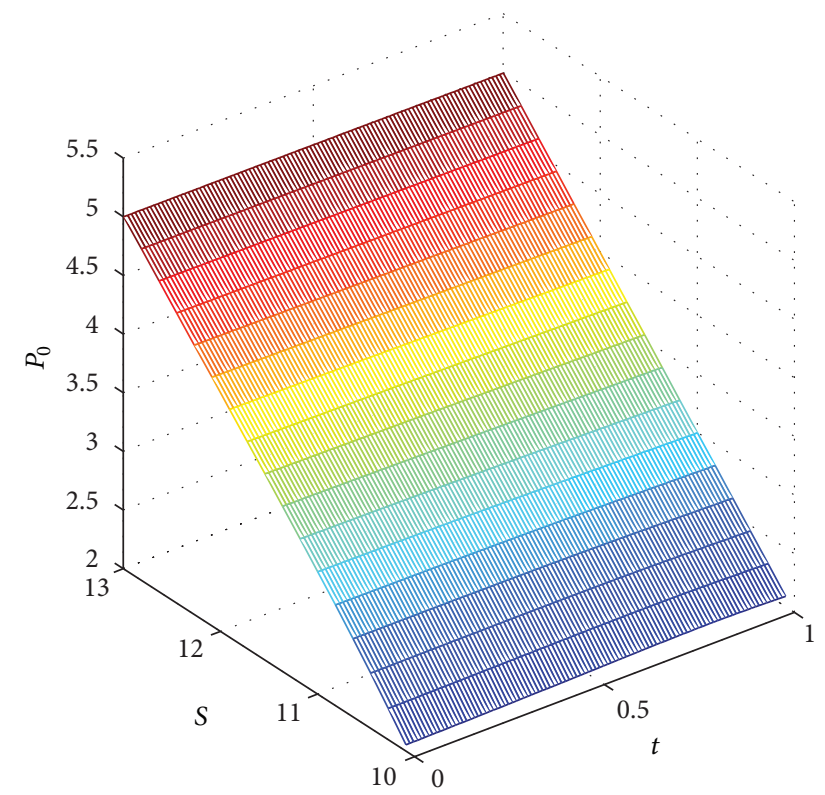

(a) $P_{0}$

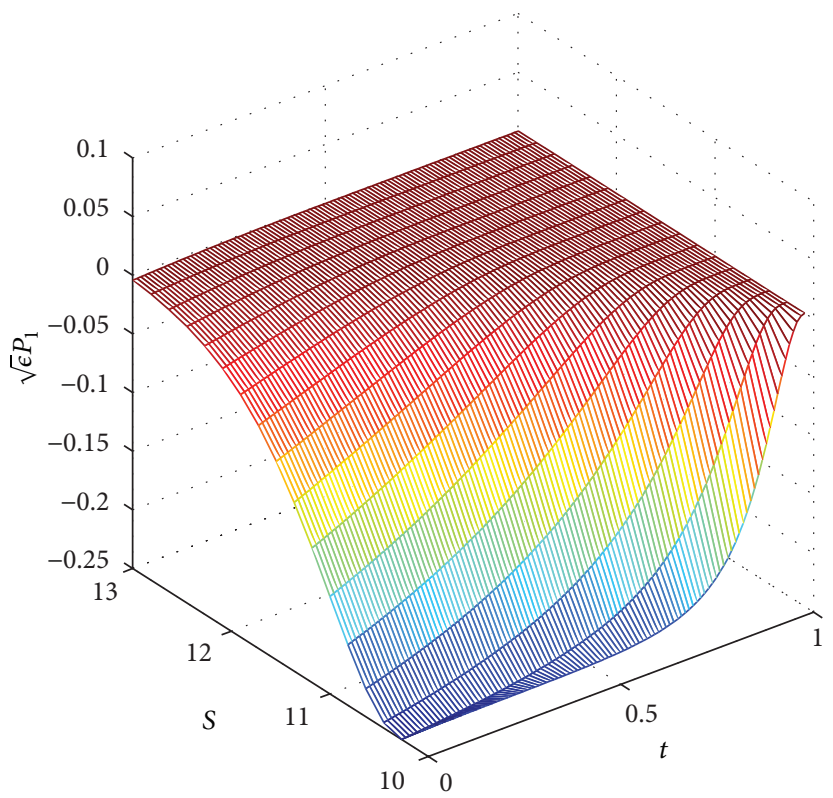

(b) $\sqrt{\epsilon} P_{1}$

FIGURE 1: The leading term and the first correction term of the turbo warrant call price is drawn under the SVCEV model with respect to time to maturity and stock price. The parameters used here are $S_{0}=10, \theta=1.8, r=0.05, q=0.03, \bar{\sigma}=0.125, T=1, T_{0}=0.2, H=9, K=8$, $W_{2}=-0.01$, and $W_{3}=0.0004$.

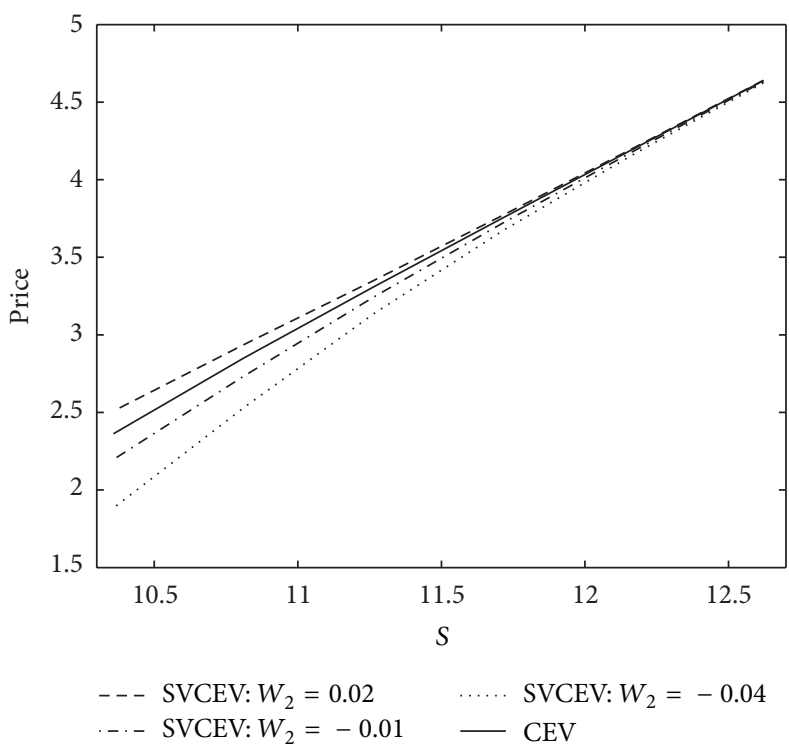

(a) $W_{3}=0.0004$

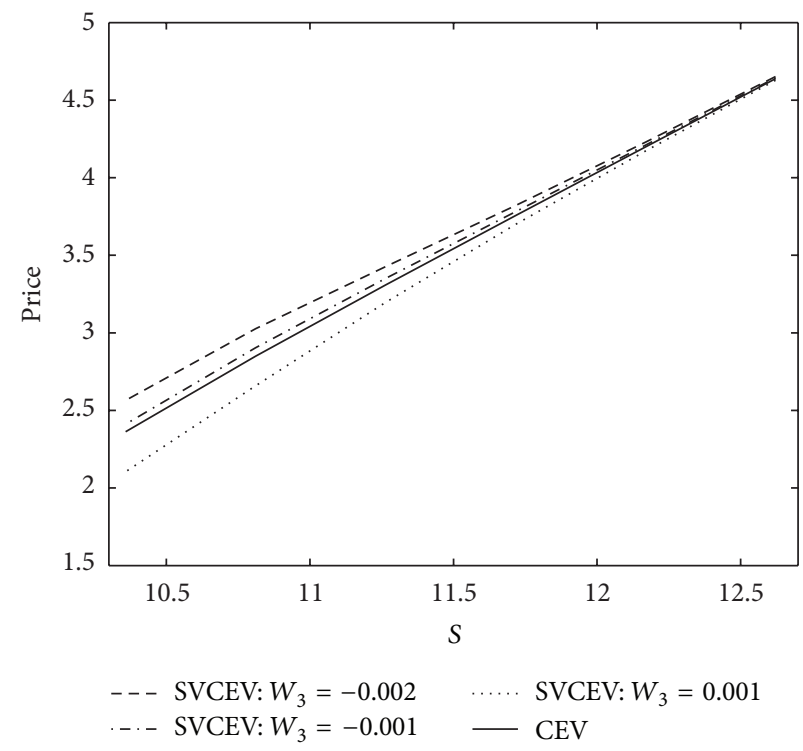

(b) $W_{2}=-0.01$

FIGURE 2: The turbo warrant call prices for different values of $W_{2}$ and $W_{3}$ under the SVCEV model are drawn. The parameters used here are $S_{0}=10, \theta=1.8, r=0.05, q=0.03, \bar{\sigma}=0.125, T=1, T_{0}=0.2, H=9$, and $K=8$.

distribution of $Y_{t}$. Further, solutions of (15) are given by the form

$$
\chi(y)=\int_{0}^{t} E^{y}\left[\phi\left(Y_{t}\right)\right] d t+\text { constant }
$$

Proof. Refer to Fouque et al. [19].

First, the leading term $P_{0}$ is obtained as follows.
Theorem 3. Under the assumed growth condition on $P_{i}$, the leading term $P_{0}$ is independent of $y$ and is the CEV price given by the solution of the PDE,

$$
\partial_{t} P_{0}+\frac{1}{2} \bar{\sigma}^{2} s \partial_{s s}^{\theta} P_{0}+(r-q) s \partial_{s} P_{0}-r P_{0}=0,
$$




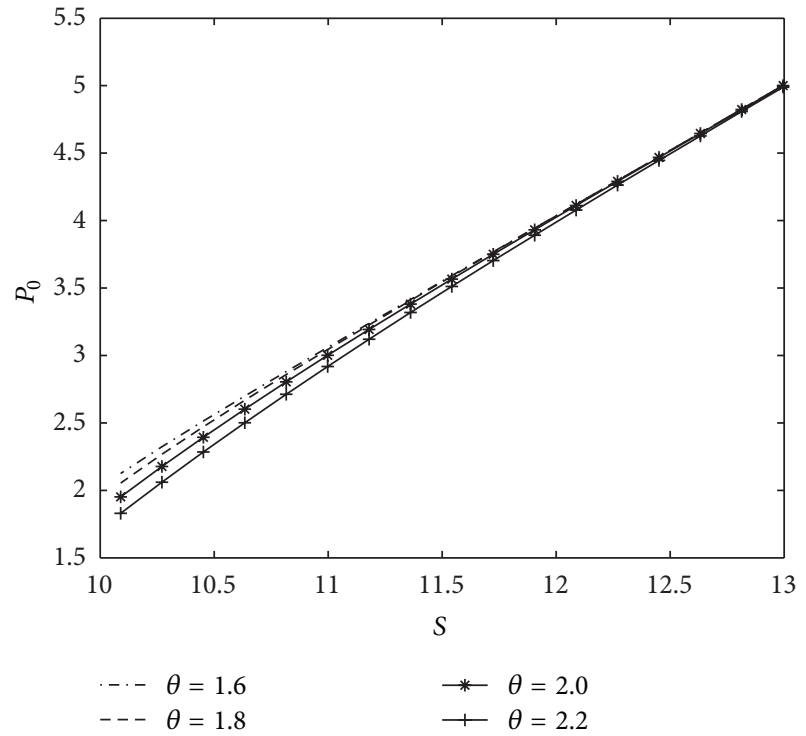

(a) $P_{0}$

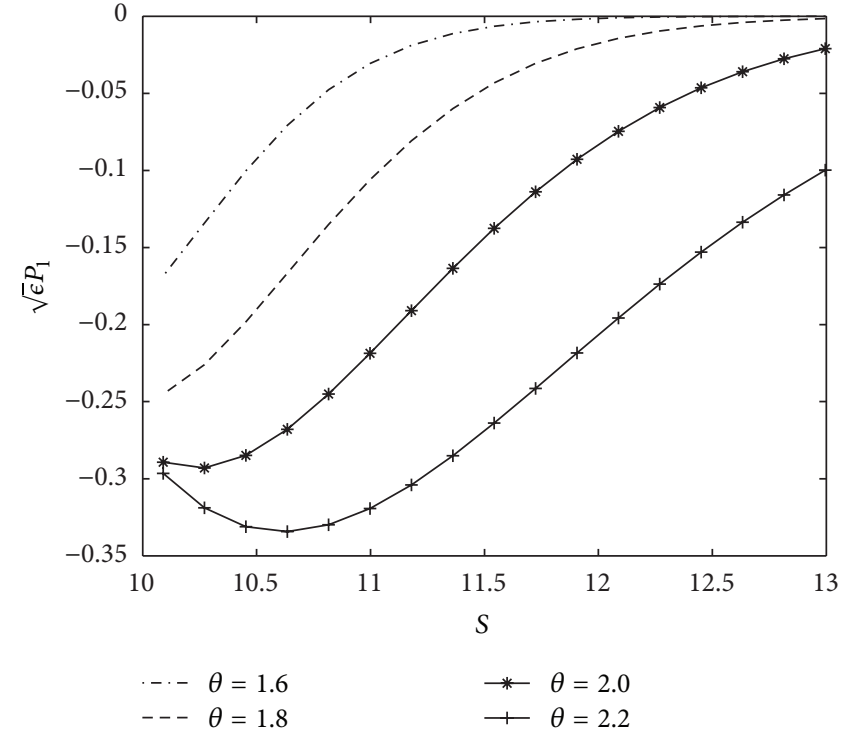

(b) $\sqrt{\epsilon} P_{1}$

FIGURE 3: The turbo warrant call prices under the CEV model and the first correction term of the SVCEV model are drawn. The parameters used here are given by $S_{0}=10, r=0.05, q=0.03, \bar{\sigma}=0.125, T=1, T_{0}=0.2, H=9, K=8, W_{2}=-0.01$, and $W_{3}=0.0004$.

with the boundary condition:

$$
\begin{gathered}
P_{0}(T, s)=s-K, \\
P_{0}\left(\tau_{H}, H\right)=L B_{0}\left(H, T_{0}\right) \\
=L C_{f l}^{0}\left(H, K, T_{0}\right)-L C_{f l}^{0}\left(H, H, T_{0}\right),
\end{gathered}
$$

where $\bar{\sigma}=\sqrt{\left\langle f^{2}\right\rangle}$.

Proof. By substituting the expansion (11) into the PDE (9), we have

$$
\begin{aligned}
\frac{1}{\epsilon} \mathscr{L}_{0} & P_{0}+\frac{1}{\sqrt{\epsilon}}\left(\mathscr{L}_{0} P_{1}+\mathscr{L}_{1} P_{0}\right) \\
& +\left(\mathscr{L}_{0} P_{2}+\mathscr{L}_{1} P_{1}+\mathscr{L}_{2} P_{0}\right) \\
& +\sqrt{\epsilon}\left(\mathscr{L}_{0} P_{3}+\mathscr{L}_{1} P_{2}+\mathscr{L}_{2} P_{1}\right)+\cdots=0,
\end{aligned}
$$

and thus the following equations are satisfied.

$$
\begin{gathered}
\mathscr{L}_{0} P_{0}=0, \quad \mathscr{L}_{0} P_{1}+\mathscr{L}_{1} P_{0}=0, \\
\mathscr{L}_{0} P_{2}+\mathscr{L}_{1} P_{1}+\mathscr{L}_{2} P_{0}=0, \\
\mathscr{L}_{0} P_{3}+\mathscr{L}_{1} P_{2}+\mathscr{L}_{2} P_{1}=0 .
\end{gathered}
$$

Then, from the growth condition applied to $\mathscr{L}_{0} P_{0}=0, P_{0}$ becomes a function of only $t$ and $s$. So, $P_{0}=P_{0}(t, s)$.

Since each term of the operator $\mathscr{L}_{1}$ contains $y$-derivative and $P_{0}$ is $y$-independent, $\mathscr{L}_{0} P_{1}+\mathscr{L}_{1} P_{0}=0$ leads to $\mathscr{L}_{0} P_{1}=0$ and so $P_{1}$ also is a function of $t$ and $s$ only; $P_{1}=P_{1}(t, s)$.

Next, $\mathscr{L}_{0} P_{2}+\mathscr{L}_{1} P_{1}+\mathscr{L}_{2} P_{0}=0$ in (20) reduces to $\mathscr{L}_{0} P_{2}+$ $\mathscr{L}_{2} P_{0}=0$ because each term of the operator $\mathscr{L}_{1}$ contains $y$-derivative and $P_{1}$ is $y$-independent. Then by Lemma 2 one has $\left\langle\mathscr{L}_{2}\right\rangle P_{0}=0$, where

$$
\left\langle\mathscr{L}_{2}\right\rangle=\partial_{t}+\frac{1}{2}\left\langle f^{2}\right\rangle s^{\theta} \partial_{s s}^{2}+(r-q) s \partial_{s}-r I,
$$

where $I$ is the identity operator. Hence, $P_{0}(t, s)$ is equal to the CEV option price with the effective volatility $\bar{\sigma}$.

Next, the term $P_{1}$ is obtained as follows.

Theorem 4. Under the assumed growth condition on $P_{i}$, the correction term $P_{1}$ is independent of $y$ and given by the solution of the PDE

$$
\begin{array}{r}
\partial_{t} P_{1}+\frac{1}{2} \bar{\sigma}^{2} s^{\theta} \partial_{s s}^{2} P_{1}+(r-q) s \partial_{s} P_{1}-r P_{1} \\
=W_{3} s^{\theta / 2} \frac{\partial}{\partial s}\left(s^{\theta} \frac{\partial^{2} P_{0}}{\partial s^{2}}\right)+W_{2} s^{\theta} \frac{\partial^{2} P_{0}}{\partial s^{2}}
\end{array}
$$

with the boundary condition:

$$
\begin{aligned}
P_{1}(T, s) & =0, \\
P_{1}\left(\tau_{H}, H\right) & =L B_{1}\left(H, T_{0}\right) \\
& =L C_{f l}^{1}\left(H, K, T_{0}\right)-L C_{f l}^{1}\left(H, H, T_{0}\right),
\end{aligned}
$$

where $W_{3}$ and $W_{2}$ are given by

$$
W_{3}=\frac{\rho v}{\sqrt{2}}\left\langle f \psi^{\prime}\right\rangle, \quad W_{2}=-\frac{v}{\sqrt{2}}\left\langle\Lambda \psi^{\prime}\right\rangle,
$$

respectively. Here, $\psi$ is defined as the solution of the Poisson equation $\mathscr{L}_{0} \psi=f^{2}-\left\langle f^{2}\right\rangle$. 


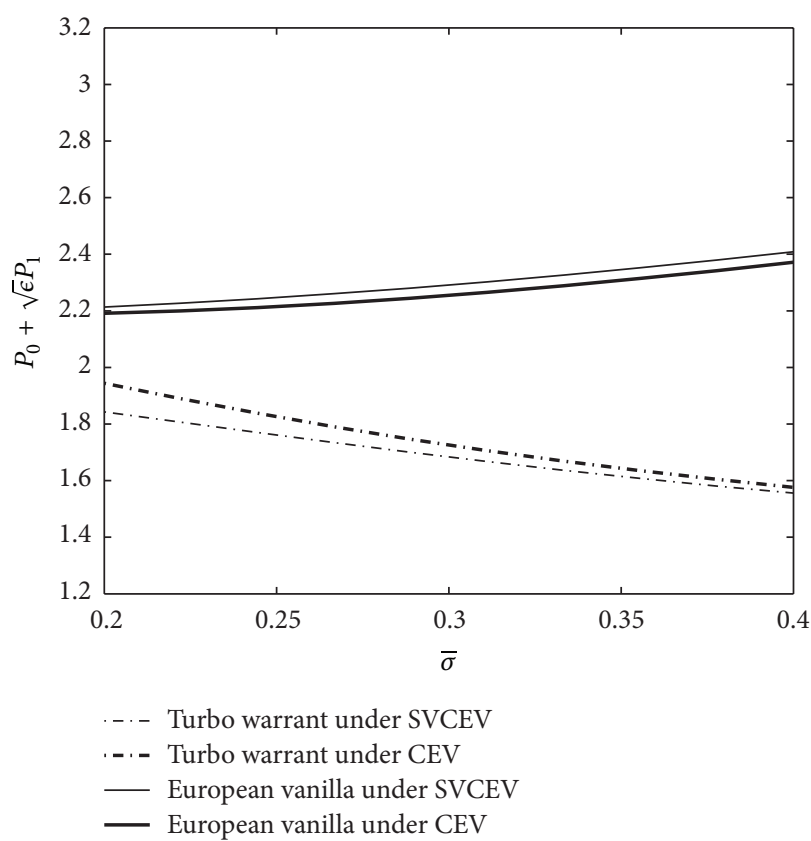

(a) $\theta=1.6$

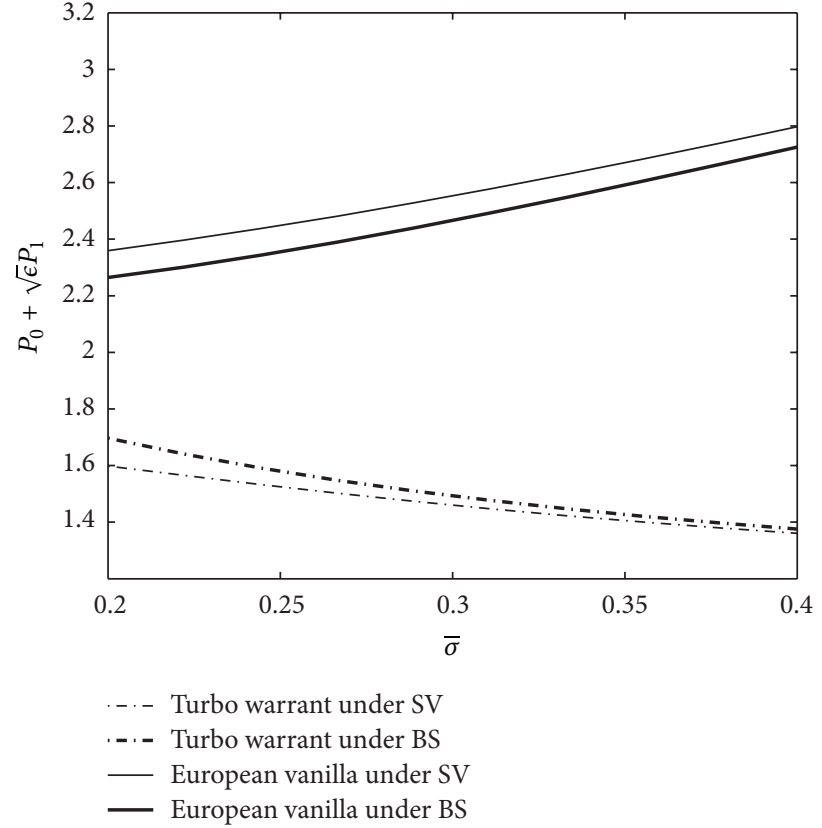

(b) $\theta=2$

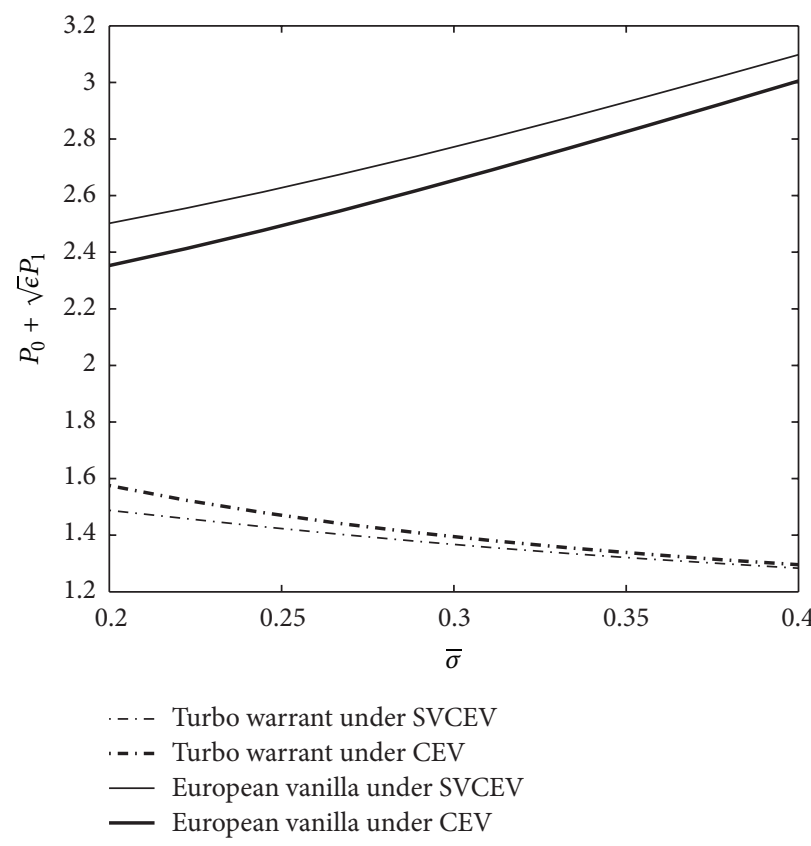

(c) $\theta=2.2$

FIGURE 4: The prices of the turbo warrant call and the European vanilla call are drawn against $\bar{\sigma}$ under the CEV and SVCEV models. The parameters used here are $S_{0}=10, r=0.05, q=0.03, T=1, T_{0}=0.2, H=9, K=8, W_{2}=-0.01$, and $W_{3}=0.0004$.

Proof. The $y$-independence of $P_{1}$ has been obtained in the proof of Theorem 3. Applying Lemma 2 to the equation $\mathscr{L}_{0} P_{3}+\mathscr{L}_{1} P_{2}+\mathscr{L}_{2} P_{1}=0$ in (20) leads to $\left\langle\mathscr{L}_{1} P_{2}+\mathscr{L}_{2} P_{1}\right\rangle=0$. Combining this one with the result $\mathscr{L}_{0} P_{2}+\mathscr{L}_{2} P_{0}=0$ obtained in the middle of proof of Theorem 3 yields the PDE (22) for $P_{1}(t, s)$.

Now, we need to solve the PDEs (17) and (22) for $P_{0}$ and $P_{1}$, respectively, for the approximation of interest. By using the result of Davydov and Linetsky [20], we obtain $P_{0}=$ $\mathrm{TC}_{\mathrm{CEV}}$ analytically as follows:

$$
\begin{aligned}
P_{0}(t, s)= & \operatorname{DOC}_{\mathrm{CEV}}(t, s) \\
& +\left[\operatorname{LC}_{\mathrm{fl}}^{\mathrm{CEV}}\left(H, K, T_{0}\right)-\operatorname{LC}_{\mathrm{fl}}^{\mathrm{CEV}}\left(H, H, T_{0}\right)\right] \\
& \times \operatorname{DR}_{\mathrm{CEV}}(t, s),
\end{aligned}
$$




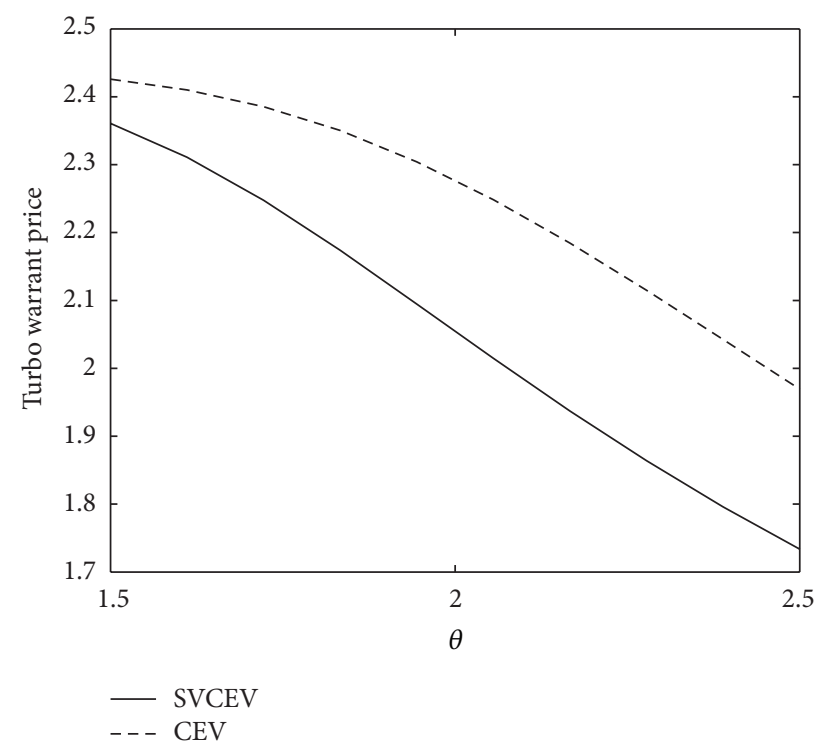

FIGURE 5: The turbo warrant call price against $\theta$ is drawn under the CEV and SVCEV models. The parameters used here are $S_{0}=10$, $r=0.05, q=0.03, \bar{\sigma}=0.125, T=1, T_{0}=0.2, H=9, K=8$, $W_{2}=-0.01$, and $W_{3}=0.0004$.

where $\mathrm{DOC}_{\mathrm{CEV}}$ is the well-known $\mathrm{CEV}$ pricing formula (cf. [20]) and $\mathrm{LC}_{\mathrm{fl}}^{\mathrm{CEV}}$ and $\mathrm{DR}_{\mathrm{CEV}}$ are given by

$$
\begin{aligned}
\operatorname{LC}_{\mathrm{fl}}\left(s, m, T_{0}\right)= & e^{-q T_{0}} \mathcal{s}-e^{-r T_{0}} m \\
& +e^{-r T_{0}} \int_{0}^{m} \mathbb{Q}\left(m_{\tau_{H}}^{T_{0}} \leq \xi\right) d \xi, \\
\operatorname{DR}_{\mathrm{CEV}}(t, s) & =E\left[e^{-r\left(\tau_{H}-t\right)} \mathbf{1}_{\left\{\tau_{H} \leq T\right\}}\right],
\end{aligned}
$$

respectively. Here, under the CEV model with the volatility coefficient $\bar{\sigma}, \mathbb{Q}\left(m_{\tau_{H}}^{T_{0}} \leq \xi\right)$ and $E\left[e^{-r\left(\tau_{H}-t\right)} \mathbf{1}_{\left\{\tau_{H} \leq T\right\}}\right]$ are given by

$$
\begin{gathered}
Q\left(m_{t}^{T_{0}} \leq \xi\right)=\mathscr{L}_{T_{0}, \lambda}^{-1}\left\{\frac{1}{\lambda} \frac{\phi_{\lambda}(H)}{\phi_{\lambda}(\xi)}\right\}, \\
E\left[e^{-r\left(\tau_{H}-t\right)} \mathbf{1}_{\left\{\tau_{H} \leq T\right\}}\right]=\mathscr{L}_{T, \lambda}^{-1}\left\{\frac{1}{\lambda} \frac{\phi_{r+\lambda}(s)}{\phi_{r+\lambda}(H)}\right\},
\end{gathered}
$$

respectively, with the Laplace transform $\mathscr{L}$, where $\lambda>0$ and

$$
\begin{gathered}
\phi_{\lambda}(s) \\
= \begin{cases}s^{\alpha+(1 / 2)} e^{(\widetilde{\theta} / 2) \tilde{x}} W_{k, \widetilde{m}}(\widetilde{x}), & \alpha<0, r-q \neq 0, \\
s^{\alpha+(1 / 2)} e^{(\widetilde{\theta} / 2) \tilde{x}} M_{k, \tilde{m}}(\widetilde{x}), & \alpha>0, r-q \neq 0, \\
s^{(1 / 2)} K_{\widetilde{\nu}}(\sqrt{2 \lambda} \widetilde{z}), & \alpha<0, r-q=0, \\
s^{(1 / 2)} I_{\tilde{\nu}}(\sqrt{2 \lambda} \tilde{z}), & \alpha>0, r-q=0,\end{cases} \\
\tilde{x}=\frac{|r-q|}{\bar{\sigma}^{2}|\alpha|} s^{-2 \alpha}, \quad \widetilde{z}=\frac{s^{-\alpha}}{\bar{\sigma}|\alpha|}, \quad \widetilde{\theta}=\operatorname{sign}(\alpha(r-q)), \\
\widetilde{m}=\frac{1}{4|\alpha|}, \quad k=\tilde{\theta}\left(\frac{1}{2}+\frac{1}{4 \alpha}\right)-\frac{\lambda}{2|\alpha(r-q)|}, \quad \widetilde{v}=\frac{1}{2|\alpha|} .
\end{gathered}
$$

Here, the functions $M_{k, m}(x)$ and $W_{k, m}(x)$ are the well-known Whittaker functions and $I_{\nu}(x)$ and $K_{\nu}(x)$ are the modified Bessel functions.

Next, the first correction term $\sqrt{\epsilon} P_{1}$ is required to be solved from the PDE (22). Apparently, it is difficult to solve (22) analytically. It requires the use of a numerical computing method. Here, we use the finite difference scheme of the Crank-Nicolson method to compute the first correction term $\sqrt{\epsilon} P_{1}$. The truncation error for the solution is $\mathcal{O}\left((\Delta t)^{2}\right)+$ $\mathcal{O}\left((\Delta s)^{2}\right)$, where $\Delta t=0.01$ and $\Delta s=0.18$.

Figure 1 shows the behavior of both the leading term and the first correction term. One can notice from the correction term that the stochastic volatility of the SVCEV model tends to lower the CEV turbo warrant price as the stock price approaches the barrier. However, the impact of the stochastic volatility decreases as time to maturity decreases. More detailed implications of the SVCEV model for the turbo warrant price are described in the following section.

From the results of Theorems 3 and 4, we formally obtain the first order approximation:

$$
P(t, s, y) \approx P_{0}(t, s)+\sqrt{\epsilon} P_{1}(t, s),
$$

where $P_{0}$ and $P_{1}$ are independent of the unobservable variable $y$. In terms of accuracy of the approximation, we note that the error estimates for barrier and lookback options have been obtained by Park and Kim [21] for the CEV model. Essentially, the required estimate for the present SVCEV model can be given by a combination of the result of [21] for the CEV model and the error estimate obtained by Fouque et al. [22] for the stochastic volatility model. The detailed proof is omitted here.

\section{Implications}

The pricing of turbo warrants under the SVCEV model is more interesting than either the CEV or stochastic volatility models. In this section, we analyze the sensitivity of the turbo warrant call option with respect to several involved parameters, in particular, the elasticity parameter $\theta$, under the SVCEV model. We note that the case of SVCEV with $\theta=2$ corresponds to the stochastic volatility (SV) model of [6] and the case of CEV with $\theta=2$ is the same as the Black-Scholes (BS) model in the following arguments.

Figure 2 plots the turbo warrant call price with respect to the parameters $W_{2}$ and $W_{3}$ under the SVCEV and the CEV price for $\theta=1.8$. As seen from (24), $W_{2}$ is a parameter related to the market price of volatility risk and $W_{3}$ is a parameter related to the correlation of the stock price and the fast-mean reverting process. It can be seen from Figure 2 that the price of the turbo warrant call increases as $W_{2}$ increases, whereas the price decreases as $W_{3}$ increases. This behavior appears more sensitively near the barrier.

Figure 3 shows that the turbo warrant call price under the CEV model and the first correction term under the SVCEV model are drawn with respect to the stock price for different values $\theta$. The stochastic volatility impact on the CEV price becomes more apparent as $\theta$ increases. It is interesting to note that the impact begins to show a nonmonotonic behavior with respect to the stock price as $\theta$ becomes larger than 2 . 


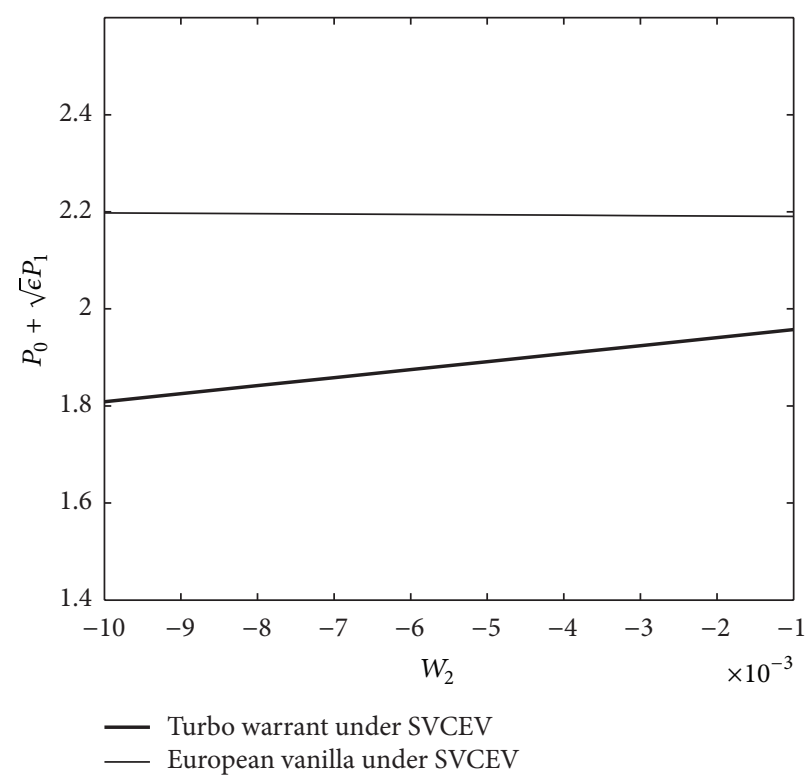

(a) $\theta=1.8$

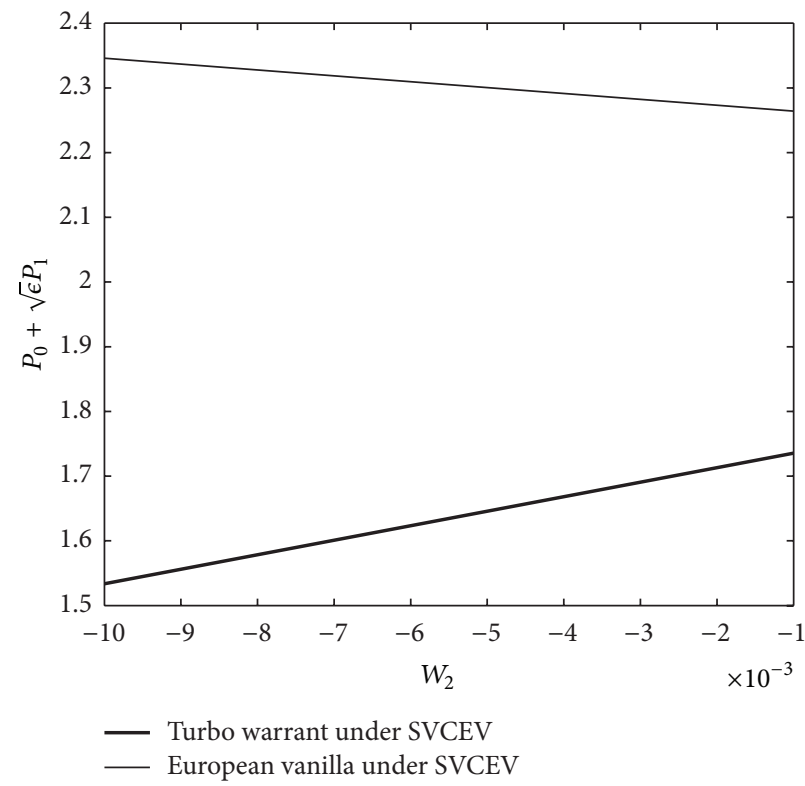

(c) $\theta=2.2$

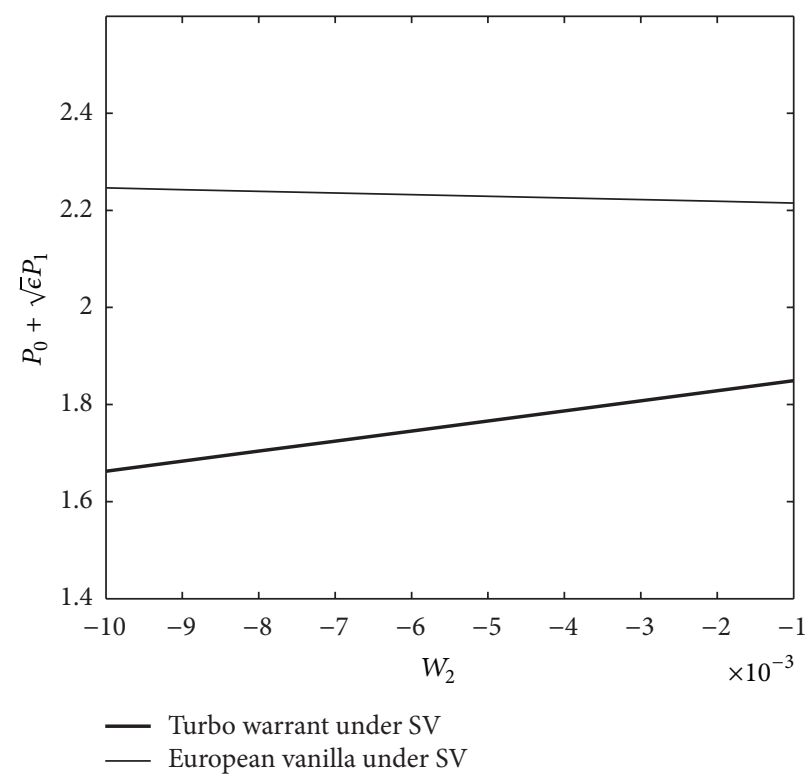

(b) $\theta=2$

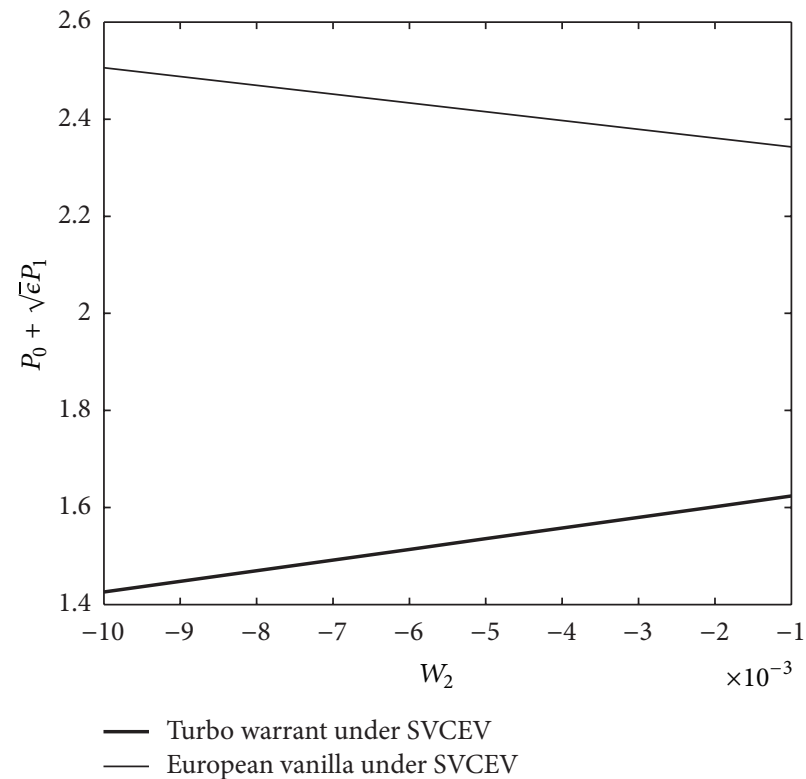

(d) $\theta=2.4$

FIGURE 6: The sensitivity of the turbo warrant call and the European vanilla call to $W_{2}$ is drawn under the SVCEV model for different values of $\theta$. The parameters used here are $S_{0}=10, r=0.05, q=0.03, T=1, T_{0}=0.2, H=9, K=8, \bar{\sigma}=0.125$, and $W_{3}=0.0004$.

Figure 4 plots the functional behavior of the European vanilla call and the turbo warrant call prices with respect to the effective volatility $\bar{\sigma}$. It is interesting to note that the European vanilla option is overpriced, whereas the turbo warrant is underpriced in the SVCEV model with respect to the CEV one. In general, the European vanilla price increases as the effective volatility $\bar{\sigma}$ rises. However, it becomes opposite for the turbo warrant case as shown in Figure 4. The turbo call price under the Black-Scholes model may not be much less sensitive to the change in volatility than the European vanilla call option as shown in [14]. But the degree of the sensitivity depends on the value of the elasticity parameter $\theta$. As shown in Figure 4, the lower $\theta$ is, the more sensitively the turbo warrant price behaves with respect to the change in volatility.

Figure 5 shows the sensitivity difference between the CEV and SVCEV models for the turbo warrant price to the change of the elasticity parameter $\theta$. Apparently, the stochastic volatility of the SVCEV model lowers the price level of the CEV turbo warrant regardless of the $\theta$ level. As $\theta$ increases, this impact tends to become larger.

Figures 6 and 7 show the sensitivity of the turbo warrant call and the European vanilla call against $W_{2}$ and $W_{3}$ under the SVCEV Model. The turbo warrant call is always cheaper 


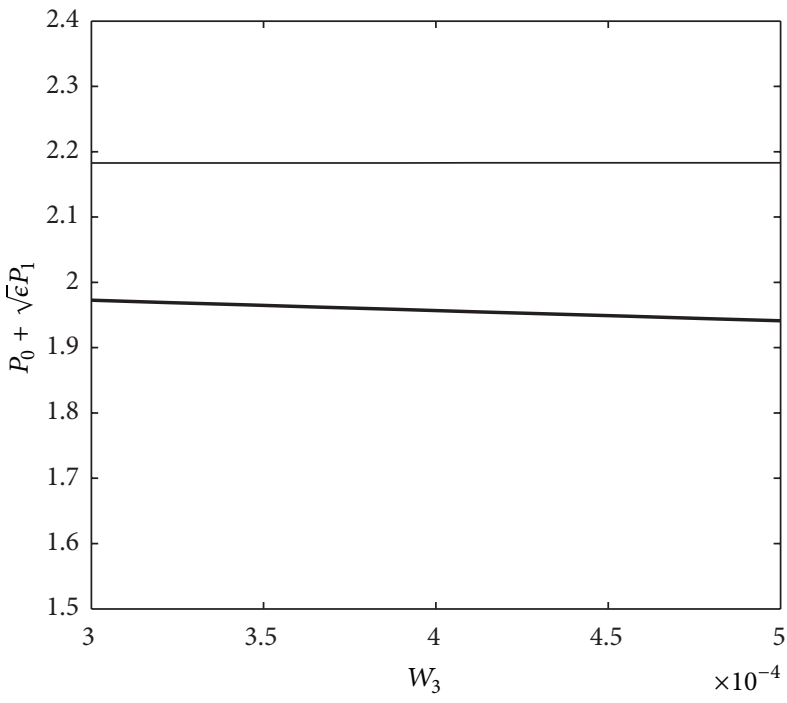

- Turbo warrant under SVCEV

— European vanilla under SVCEV

(a) $\theta=1.6$

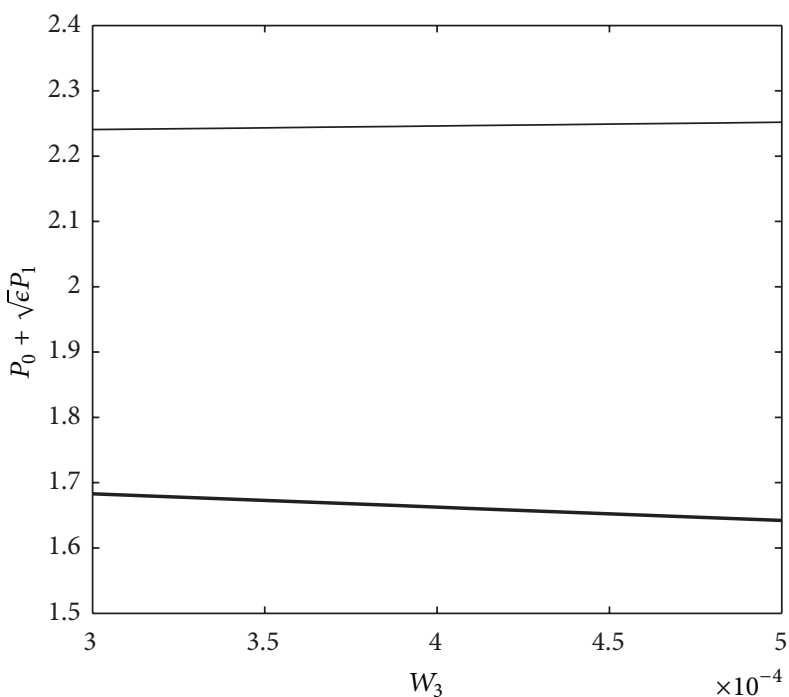

— Turbo warrant under SV

_ European vanilla under SV

(c) $\theta=2$

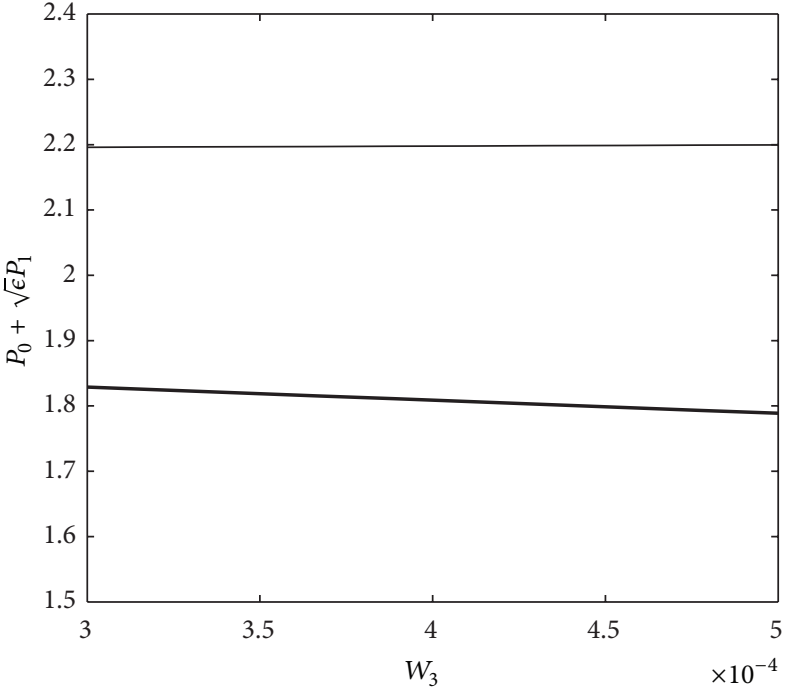

Turbo warrant under SVCEV

— European vanilla under SVCEV

(b) $\theta=1.8$

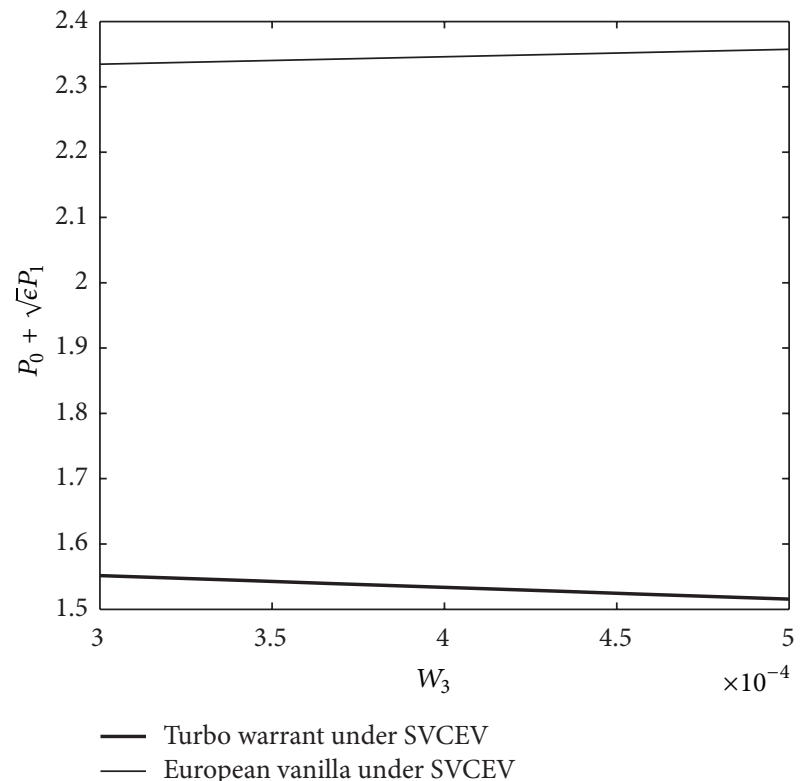

(d) $\theta=2.2$

FIgURE 7: The sensitivity of the turbo warrant call and the European vanilla call to $W_{3}$ is drawn under the SVCEV model. The parameters used here are $S_{0}=10, r=0.05, q=0.03, T=1, T_{0}=0.2, H=9, K=8, \bar{\sigma}=0.125$, and $W_{2}=-0.01$.

than the European vanilla call as expected. Figure 6 shows that as $W_{2}$ increases, the price of the turbo warrant call increases, whereas the price of the vanilla call decreases. The European vanilla call is more sensitive to $W_{2}$ than the turbo warrant as $\theta$ increases. Figure 7 shows that the price of the turbo warrant call drops moderately while the price of the vanilla call rises very slowly as $W_{3}$ increases.

\section{Conclusion}

In this paper, we have examined the price change behavior of a turbo warrant with respect to the involved parameters (effective volatility, elasticity, the market price of volatility risk, and the correlation between stock price and volatility) under the SVCEV model. The hybrid nature of the model enables us to find the rich sensitivity structure of the turbo warrant price in various ways depending upon the stochastic volatility as well as the elasticity of variance. This paper not only shows the stochastic volatility effect on the CEV price of the turbo warrant but also the impact of the elasticity of variance on the stochastic volatility price. Moreover, a comparison analysis of the turbo warrant contract and the European vanilla option has been performed effectively based on the hybrid model. 


\section{Conflict of Interests}

The authors declare that there is no conflict of interests regarding the publication of this paper.

\section{Acknowledgments}

The authors would like to thank the anonymous referees for their valuable comments and suggestions to improve the paper. The research of Jeong-Hoon Kim was supported by the National Research Foundation of Korea NRF2013R1A1A2A10006693. The research of Min-Ku Lee was supported by BK21 PLUS Sungkyunkwan University Mathematics Division and the research of Ji-Hun Yoon was supported by BK21 PLUS SNU Mathematical Sciences Division.

\section{References}

[1] J. Eriksson, On the pricing equations of some path-dependent options [Ph.D. dissertation], Uppsala University, Uppsala, Sweden, 2006.

[2] F. Black and M. Scholes, "The pricing of options and corporate liabilities," Journal of Political Economy, vol. 81, pp. 637-654, 1973.

[3] J. Cox, "Notes on option pricing I: constant elasticity of variance diffusions," Working Paper, Stanford University, 1975, reprinted in The Journal of Portfolio Management, vol. 22, pp. 15-17, 1996.

[4] J. C. Cox and S. A. Ross, "The valuation of options for alternative stochastic processes," Journal of Financial Economics, vol. 3, no. 1-2, pp. 145-166, 1976.

[5] S. L. Heston, "Closed-form solution for options with stochastic volatility with applications to bond and currency options," The Review of Financial Studies, vol. 6, pp. 327-343, 1993.

[6] J.-P. Fouque, G. Papanicolaou, and K. R. Sircar, "Asymptotics of a two-scale stochastic volatility model," in Equations Aux Derivees Partielles et Applications, Articles Dedies a JacquesLouis Lions, pp. 517-525, Gauthier-Villars, Paris, France, 1998.

[7] P. Carr, H. Geman, D. B. Madan, and M. Yor, "Stochastic volatility for Lévy processes," Mathematical Finance, vol. 13, no. 3, pp. 345-382, 2003.

[8] P. S. Hagan, D. Kumar, A. S. Lesniewski, and D. E. Woodward, Managing Smile Risk, Wilmott Magazine, 2002.

[9] S.-Y. Choi, J.-P. Fouque, and J.-H. Kim, "Option pricing under hybrid stochastic and local volatility," Quantitative Finance, vol. 13, no. 8, pp. 1157-1165, 2013.

[10] B.-H. Bock, S.-Y. Choi, and J.-H. Kim, "The pricing of European options under the constant elasticity of variance with stochastic volatility," Fluctuation and Noise Letters, vol. 12, no. 1, Article ID 135004, 13 pages, 2013

[11] M. -K. Lee, J. -H. Kim, and K. -H. Jang, "Pricing arithmetic Asian options under hybrid stochastic and local volatility," Journal of Applied Mathematics, Article ID 784386, 2014.

[12] H. Y. Wong and C. M. Chan, "Turbo warrants under stochastic volatility," Quantitative Finance, vol. 8, no. 7, pp. 739-751, 2008.

[13] A. Domingues, The valuation of turbo warrants under the CEV Model [M.S. thesis], ISCTE Business School, University Institute of Lisbon, Lisbon, Portugal, 2012.

[14] H. Y. Wong and K. Y. Lau, "Analytical valuation of turbo warrants under double exponential jump diffusion," Journal of Derivatives, vol. 15, no. 4, pp. 61-73, 2008.
[15] G. Tataru and F. Travis, Stochastic Local Volatility, Quantitative Development Group, Bloomberg, 2010, version 1.

[16] J.-H. Kim, J. Lee, S.-P. Zhu, and S.-H. Yu, "Amultiscale correction to the Black-Scholes formula," Applied Stochastic Models in Business and Industry. In press.

[17] B. Øksendal, Stochastic Differential Equations, Springer, New York, NY, USA, 6th edition, 2003.

[18] H. Y. Wong and C. M. Chan, "Lookback options and dynamic fund protection under multiscale stochastic volatility," Insurance, vol. 40, no. 3, pp. 357-385, 2007.

[19] J.-P. Fouque, G. Papanicolaou, R. Sircar, and K. Sølna, Multiscale Stochastic Volatility for Equity, Interest Rate, and Credit Derivatives, Cambridge University Press, Cambridge, UK, 2011.

[20] D. Davydov and V. Linetsky, "Pricing and hedging pathdependent options under the CEV process," Management Science, vol. 47, no. 7, pp. 949-965, 2001.

[21] S.-H. Park and J.-H. Kim, "Asymptotic option pricing under the CEV diffusion," Journal of Mathematical Analysis and Applications, vol. 375, no. 2, pp. 490-501, 2011.

[22] J.-P. Fouque, G. Papanicolaou, R. Sircar, and K. Solna, "Singular perturbations in option pricing," SIAM Journal on Applied Mathematics, vol. 63, no. 5, pp. 1648-1665, 2003. 


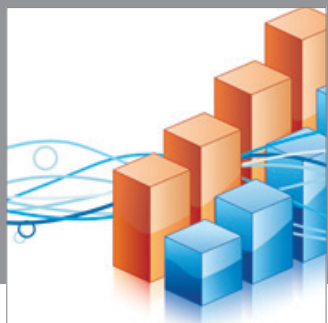

Advances in

Operations Research

mansans

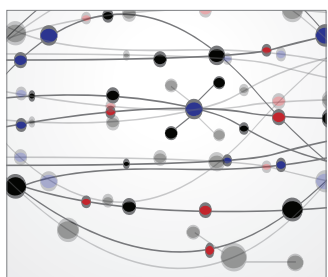

The Scientific World Journal
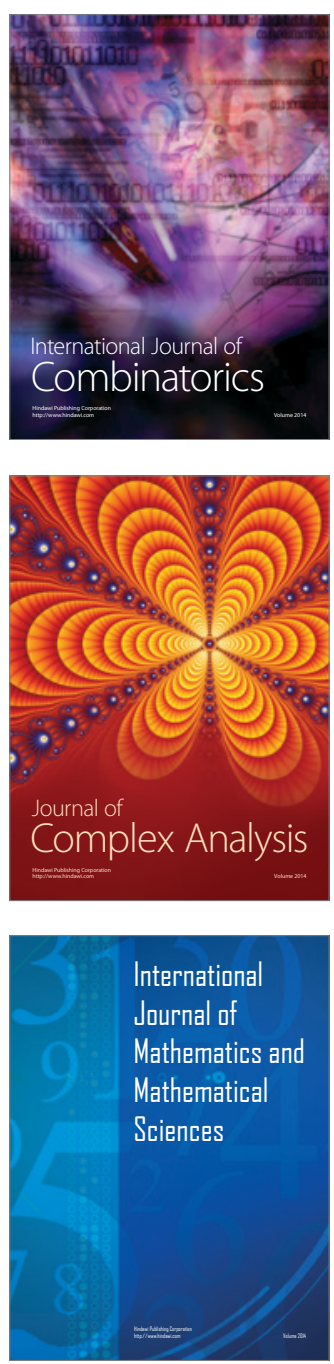
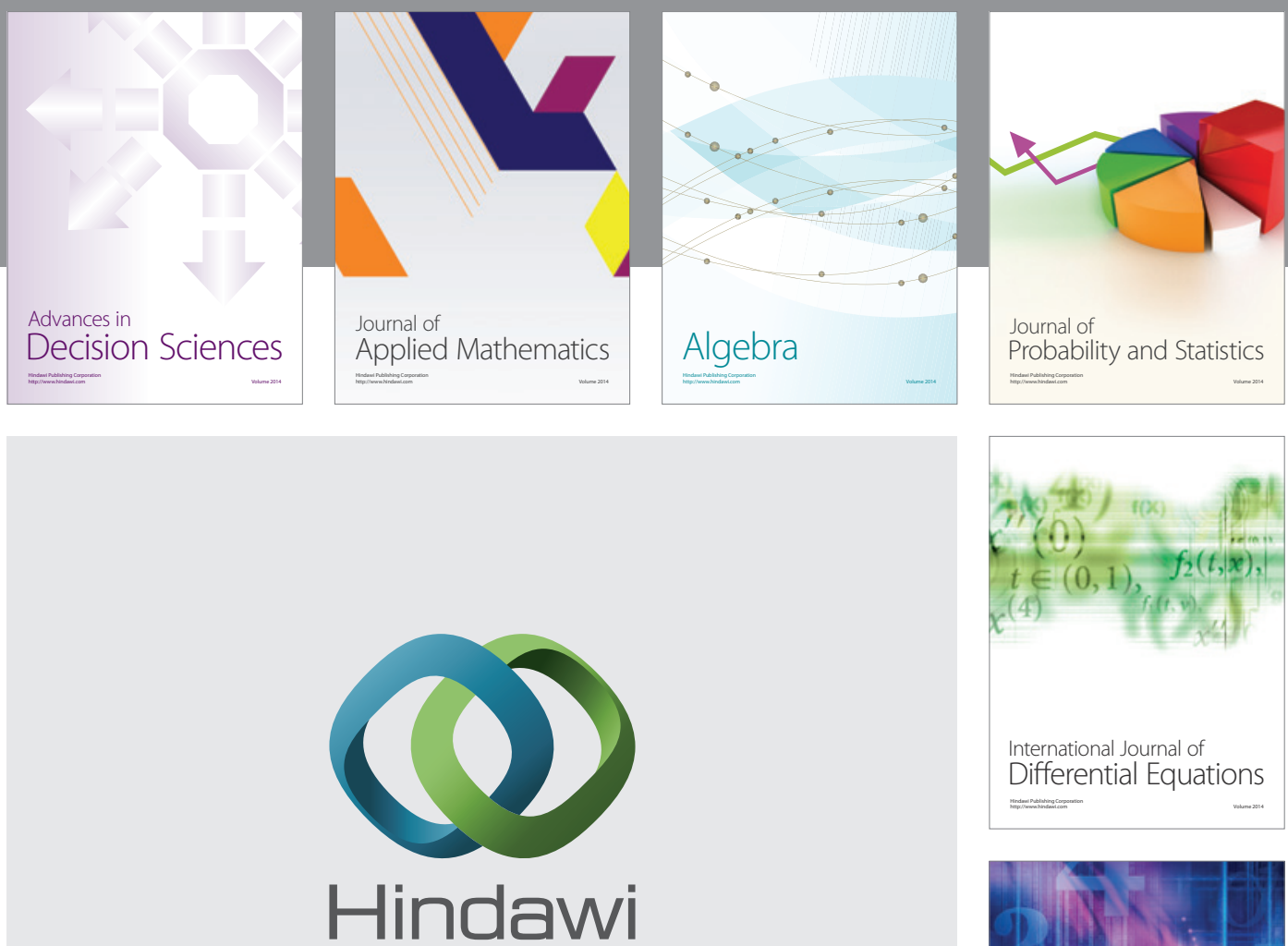

Submit your manuscripts at http://www.hindawi.com
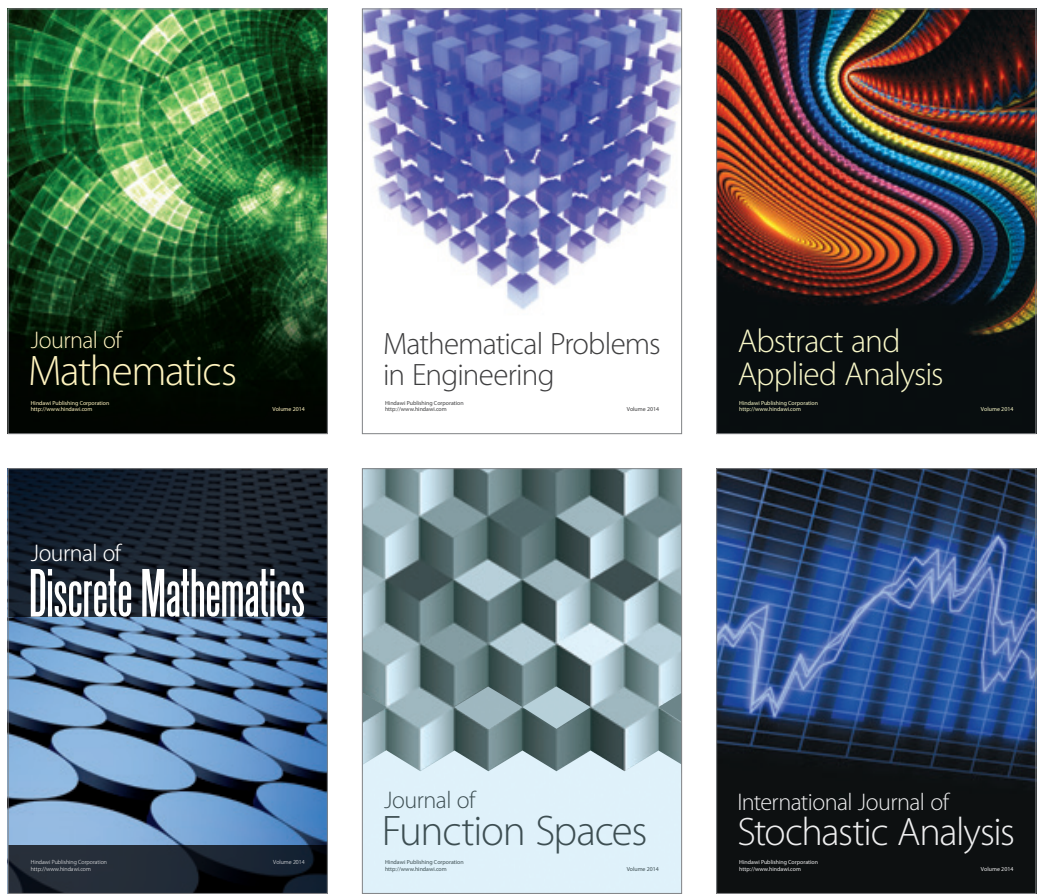

Journal of

Function Spaces

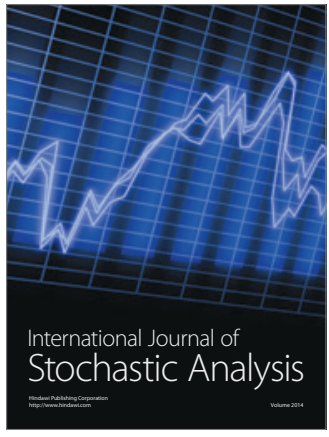

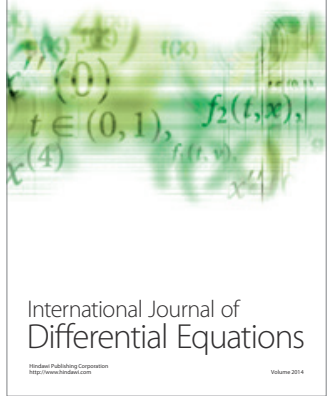
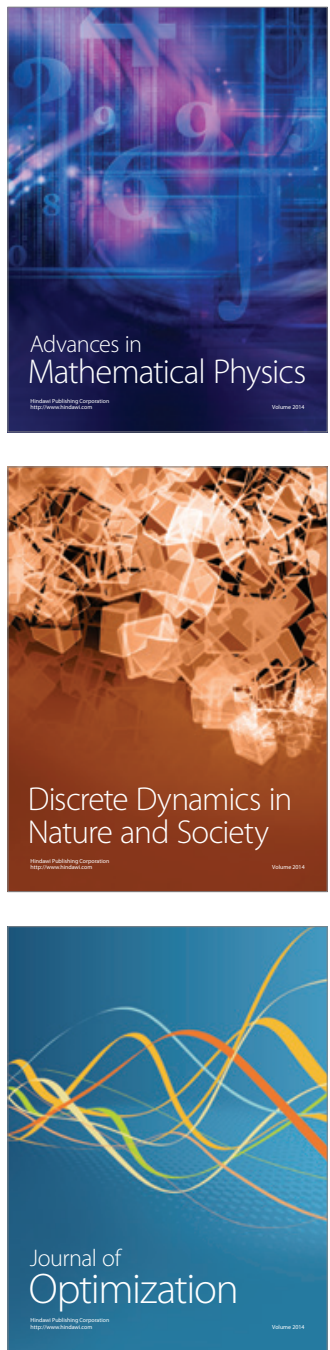